\title{
Laparoscopic Assisted Orchidectomy Eases Future Insertion of Testicular Prosthesis in Paediatric Patients
}

\author{
Dayang Anita Abdul Aziz*1 \\ Zainal Adwin Abidin ${ }^{1}$ \\ Mahmud Mohd Nor ${ }^{2}$ \\ Suria Hayati Md Pauzi ${ }^{2}$ \\ Rahmah Rasat ${ }^{3}$ \\ Zarina Abdul Latiff ${ }^{3}$
}

\author{
'Paediatric Surgery Unit \\ Department of Surgery \\ UKM Medical Centre, Malaysia. \\ ${ }^{2}$ Department of Pathology \\ UKM Medical Centre, Malaysia. \\ ${ }^{3}$ Department of Paediatrics \\ UKM Medical Centre, Malaysia.
}

Correspondence to:

Dr. Dayang Anita Abdul Aziz

Associate Professor and Head of Paediatric Surgery UKM Medical Centre

Jalan Yaacob Latif, Bandar Tun Razak

Cheras, Kuala Lumpur

56000 MALAYSIA

Email: dayanganita@yahoo.co.uk

Phone: +60123059439

Fax: +603 91456684

$w w w . b j$ e $n d o$. org

\begin{abstract}
High ligation orchidectomy in paediatric patients is performed for testicular tumours. This is carried out via open surgery at the inguinal or groin region. In these boys, elective insertion of testicular prostheses is carried out later to improve the external genitalia appearance. In most cases, insertion of testicular prosthesis or implant is carried out via the previous scar, to avoid prosthesis extrusion; however this is usually difficult due to scarring and may cause haematoma and possible infection. We report a novel technique of laparoscopic assisted orchidectomy in an adolescent boy with disorder of sexual development (DSD) whom was suspected of having bilateral gonadal (testicular) malignant change, he successfully underwent bilateral ligation of testicular vessels laparoscopically and removal of both testes via a midline scrotal raphe incision; hence avoiding bilateral groin incisions. With this method, future insertion of testicular prostheses can be carried out via virgin inguinal incisions.
\end{abstract}

Key words: Orchidectomy; laparoscopic assisted; paediatric; testicular prostheses.

\section{INTRODUCTION}

The standard treatment of paediatric patients with testicular tumours is to undergo radical inguinal orchidectomy and high ligation of spermatic vessels regardless of the types of testicular tumour ${ }^{1,2,3}$. Scrotal orchidectomy is also performed by some centres but stand the risk of scrotal violation and incomplete resection ${ }^{1,2}$. Laparoscopic orchidectomy is performed for straightforward intraabdominal testis which looks abnormal or has become gangrenous from torsion ${ }^{4}$. We share our experience at managing an adolescent patient with disorder of sexual differentiation (DSD) who required bilateral orchidectomy. The technique was simple and complied to the principle of high ligation of spermatic vessels and intact testicular removal.

\section{CASE REPORT}

A thirteen year old boy, a known 46XX with ovotesticular DSD and was referred to us for suspicious enlargement of both testes. He was born termed with no dysmorphism but with ambiguous genitalia. Clinical assessment and investigation during infancy revealed a phenotypically male baby with penoscrotal hypospadias, bifid scrotum, micropenis, palpable right testis and impalpable left testis. The chromosomal analysis revealed karyotype 46XX. He was brought up as a male. A laparoscopic examination and testicular biopsy of the intra-abdominal left testis was also carried out and confirmed the presence of ovotesticular tissue. However the left testis was not brought down. He underwent staged hypospadias repair between ages 3 to 5 years old. He was noted to have developmental delay and was attending special school. He was also on testosterone hormonal therapy.

At the current presentation, on examination, he was a small size boy with speech and language impairment. He could speak but with much difficulty. He was able to understand simple instructions. According to the mother, his son was able to tend to himself most of the times and was also toilet trained. He had palpable breast tissue bilaterally (Tanner Stage II). Genital examination revealed a small penis about $3 \mathrm{~cm}$ in length; urethral meatus was at the tip and a well-developed 
scrotum. Both testes were palpabled in the scrotum. The right testis was bigger than left and the size was $3.5 \mathrm{X}$ $2.0 \mathrm{~cm}$, firm to hard in consistency. The left testis was flattened shaped, rather soft and flaccid in consistency; about 2 X $2 \mathrm{~cm}$. Ultrasound of both testes revealed homogenous testicular echogenicity bilaterally with no other abnormality. An MRI of the abdomen revealed a blind ended uterus without fallopian tubes and no pelvic or retroperitoneal lymph nodes enlargement. The abnormal enlarged, hard right testis raised the suspicion of gonadal tumour with malignant change i.e. gonadoblastoma or even benign tumours like cystadenomas; whereas the flattened left testis was also likely abnormal or dysgenetic. Decision was made to remove both testes, parents were counselled for laparoscopic assessment of pelvic structures and laparoscopic assisted bilateral orchidectomy. Parents also agreed for elective insertion of testicular prostheses once they were able to afford the prostheses.

Surgery was done under general anaesthesia. Patient was positioned supine and the abdomen and perineum were cleaned and draped. A stab supraumbilical incison was made and peritoneal cavity was entered in layers. The camera port was a $6 \mathrm{~mm}$ Hasson. Pneumoperitoneum was created using pressure of $12 \mathrm{mmHg}$ and $\mathrm{CO} 2$ insufflation started at gas flow rate of 0.5 litre/ minute to 3 litre/minute. Two working instruments (Kelly's forceps) were introduced via port-free technique as patient's abdominal wall is quite thin. Interestingly, this patient did not have vas deferens on both sides (Figure 1 and Figure 2). Only a streak uterus was seen with no fallopian tubes. Cauterization of the spermatic vessels was carried out with ease bilaterally at the level of the internal ring. The pneumoperitoneum was maintained whilst the testes were removed. Externally, an incision was made at the scrotal midline raphe, the right testis was identified first, detached from the gubernaculum and surprisingly it was not difficult to pull out the right testis with the tunica albuginea remained intact; bipolar diathermy was used for dissection caudally. The same procedure was carried out for the left testis. The midline scrotal raphe wound was closed in layers and cyanoacrylate (tissue glue) was used on the skin (Figure 3). The laparoscopic appearance post completion of orchidectomy confirmed that haemostasis was secured (Figure 4 and Figure 5) Closure of abdominal wounds was also performed using cyanoacrylate tissue glue. Local infiltration of lignocaine was given to the wounds as analgesia.

Post-operatively, patient was well and pain- free. He was discharged home the same evening. Both testes were sent fresh to our pathologist. The histopathology examination revealed presence of testicular and ovarian tissues in each testis with no signs of malignancy. The patient remained well and is due for his first implant in 3 months time.

\section{DISCUSSION}

In this particular patient, the laparoscopic assisted bilateral orchidectomy was chosen to avoid bilateral inguinal incisions which may have caused more pain, which may also lead to scarring and future difficulty at inserting the testicular prostheses. The authors' technique still adheres to the correct principle of high ligation of testicular vessels when anticipating tumour-laden testes. Insertion of testicular prostheses is safest using groin incision to avoid prosthesis extrusion ${ }^{2,}{ }^{3}$. Insertion of prosthesis at the same time as inguinal orchidectomy is not carried out by the authors for two main reasons; firstly the testicular prosthesis is not readily available in their hospital, it has to be ordered and usually take quite a few weeks of planning. Secondly the prosthesis has to be paid by the patient's parent(s) or caretaker. The price of prosthesis is quite expensive as such the authors prefer to insert the prosthesis only once at patient's age consistent to Tanner Stage IV or $\mathrm{V}$ of Development i.e. about 15-18 years old, this way the adult-size testicular prosthesis can be used; unless the parents or caretaker can afford to pay for multiple replacement prostheses. In this patient, the final histology did not show any tumour cells with malignant change. Patients with ovotesticular DSD has $2.6 \%$ chance of having gonadal tumours with malignant potential; although more seen in 46XY patients ${ }^{5}$. Nevertheless based on the clinical presentation, it was not possible to rule this out. Laparoscopic assisted bilateral orchidectomy was indeed a feasible and simple technique in this patient. This technique has never been described before; perhaps it should be considered by others in the future when faced with similar situations.

\section{DISCLOSURE}

All the authors declared no competing interest.

\section{References}

1. K. Maguire, R. Carachi. Testicular Tumour. Basic Techniques in Pediatric Surgery 2013; F7: 396-7. DOI: 10.1007/978-3-642-20641-2-120.

2. Lawrentschuk N, Webb DR. Insertion testicular prostheses: a new surgical technique for difficult cases. BJU Int 2005; 95(7): 1111-4.

3. D Bodiwala, DJ Summerton, TR Terry. Testicular prostheses: development and modern usage. Ann R Coll Surg Engl 2007; 89: 349-53. DOI: 10.1308/003588407X183463.

4. Lee KF, Tang YC, Leong HT. Emergency laparoscopic orchidectomy for torsion of intra-abdominal testis: a case report. J R Coll Surg Edinb 2001; 46(2):110-2.

5. M Dayal et al. Ovotesticular Disorder of Sexual Development. http://emedicine.medscape.com/ article/ 256289-overview. 